\title{
Two intermediate incommensurate phases in the molecular dissociation process of solid iodine under high pressure
}

\author{
Hiroshi Fujihisa $\odot,{ }^{1, *}$ Kenichi Takemura $\odot,{ }^{2,3}$ Mitsuko Onoda, ${ }^{2}$ and Yoshito Gotoh $\odot^{1}$ \\ ${ }^{1}$ National Metrology Institute of Japan (NMIJ), National Institute of Advanced Industrial Science and Technology (AIST), \\ Tsukuba Central 5, Tsukuba, Ibaraki 305-8565, Japan \\ ${ }^{2}$ National Institute for Materials Science (NIMS), Tsukuba, Ibaraki 305-0044, Japan \\ ${ }^{3}$ Institute of Materials Structure Science (IMSS), High Energy Accelerator Research Organization (KEK), \\ Tsukuba, Ibaraki 305-0801, Japan
}

(Received 27 May 2020; revised 7 July 2021; accepted 9 July 2021; published 20 August 2021)

\begin{abstract}
A high-pressure phase of solid iodine is identified in the pressure range of $16-23 \mathrm{GPa}$ at room temperature by reanalyzing the previous powder x-ray diffraction data. The phase VI has a four-dimensional (4D) incommensurately modulated structure, in which $\mathrm{I}_{2}$ molecules and zigzag chains of three iodine atoms coexist. This structural model can successfully explain the Raman scattering data previously reported. Furthermore, our method clarifies that the structure of phase $\mathrm{V}$, which was once analyzed as a 4D incommensurately modulated structure, can be better described by a five-dimensional incommensurately modulated tetragonal lattice wherein two modulation waves run in orthogonal directions. Our findings show that the pressure-induced molecular dissociation proceeds in multiple stages that are more complex than previously thought. In addition, it has become necessary to describe the molecular dissociation pressure separately for the starting pressure $P_{s}$ of $16 \mathrm{GPa}$ and the finishing pressure $P_{f}$ of $29 \mathrm{GPa}$. This transition process has finally resolved the contradiction with the Mössbauer experiment.
\end{abstract}

DOI: 10.1103/PhysRevResearch.3.033174

\section{INTRODUCTION}

Solid halogens are representative diatomic molecular crystals in which halogen molecules are weakly bound by the van der Waals force. The effect of high pressure is interesting because pressure forces molecules to approach each other, thereby significantly modifying the intermolecular interactions. Two major changes can be expected for such a densely packed molecular crystal: metallization and molecular dissociation. Iodine has been the element most intensively investigated because it shows these phenomena at relatively low pressures.

Early high-pressure experiments reported that the electrical resistance of iodine gradually decreases with pressure, and iodine becomes metallic $>16 \mathrm{GPa}$ at room temperature [1-3]. A later x-ray diffraction study up to $20.6 \mathrm{GPa}$ [4] showed that iodine undergoes no structural phase transition during metallization, keeping the same molecular structure as that of phase I (orthorhombic space group Cmce [5,6]), the stable form of solid iodine under ambient conditions. Subsequent experiments at higher pressures revealed that iodine undergoes a structural phase transition called molecular dissociation [7] to a nonmolecular (monatomic) phase (phase II) at $\sim 21 \mathrm{GPa}$ [8-10]. In other words, iodine first becomes metallic with-

\footnotetext{
*hiroshi.fujihisa@aist.go.jp

Published by the American Physical Society under the terms of the Creative Commons Attribution 4.0 International license. Further distribution of this work must maintain attribution to the author(s) and the published article's title, journal citation, and DOI.
}

out any accompanying structural change and then undergoes pressure-induced molecular dissociation at higher pressures [9]. Further experiments revealed that phase II successively transforms to phases III and IV at 43 and $55 \mathrm{GPa}$, respectively $[11,12]$. Shimizu et al. $[13,14]$ found a superconducting state of iodine at $28 \mathrm{GPa}$ and at $1.2 \mathrm{~K}$ by electrical resistance and magnetic susceptibility measurements, where iodine is in metallic monatomic phase II. In addition to these nonmolecular phases, phase $\mathrm{V}$ has been found in the pressure range 22-28 GPa in later experiments [15]. Phase V has an incommensurate modulated structure, which can be viewed as an intermediate phase between molecular and nonmolecular ones. Independent Raman-scattering experiments clarified that the Raman spectra of iodine $>23 \mathrm{GPa}$ has a mode characteristic of modulated structures and supported the existence of phase V [16]. The upper side of Table I summarizes the crystal structures for all reported phases of iodine.

Although the behavior of iodine under high pressure seems to be well understood, a question remains as to whether iodine undergoes any structural phase transitions in the molecular phase before the dissociation. Mössbauer spectroscopy experiments up to $30 \mathrm{GPa}$ suggested the coexistence of a quasi-one-dimensional (quasi-1D) structure (HP1) $>16 \mathrm{GPa}$ and a quasi-two-dimensional (quasi-2D) structure (HP2) $>21$ $\mathrm{GPa}$ and claimed that a molecular character remains up to $30 \mathrm{GPa}$ [17]. Raman-scattering experiments also reported bands appearing $>10 \mathrm{GPa}$, suggesting the existence of an intermediate molecular phase [16,18].

In contrast to these experiments, x-ray diffraction studies showed no evidence of structural phase transitions in molecular phase I of iodine. Although we noticed that the measured peak positions of the diffraction patterns of phase I 
TABLE I. Crystal structures of the high-pressure phases of iodine at room temperature before (upper) and after (lower) this study. Phase VI was identified in the pressure range 16-23 GPa. The structure of phase V was reanalyzed by a 5D superspace group. The revisions are shown in red. The molecular dissociation starts at pressure $P_{s}=16 \mathrm{GPa}$ and finishes at $P_{f}=29 \mathrm{GPa}$ (see text).

\begin{tabular}{|c|c|c|c|}
\hline \multicolumn{4}{|c|}{ Phase diagram of iodine at room temperature } \\
\hline Phase & $P(\mathrm{GPa})$ & Crystal system & Space group \\
\hline \multicolumn{4}{|c|}{ Before this study } \\
\hline I & $0-24$ & Orthorhombic & $\mathrm{Cmce}^{\mathrm{a}}[5]$ \\
\hline $\mathrm{V}$ & $24-29$ & 4D orthorhombic (incommensurately modulated) & $F m m m(\alpha 00) 0 s 0[19]$ \\
\hline II & $29-43$ & Orthorhombic & Immm [8] \\
\hline III & $43-55$ & Tetragonal & $I 4 / \mathrm{mmm}[11]$ \\
\hline IV & $55-(276)$ & Cubic & $F m-3 m[12,20]$ \\
\hline \multicolumn{4}{|c|}{ After this study } \\
\hline I & $0-16$ & Orthorhombic & Cmce $^{\mathrm{a}}[5]$ \\
\hline VI & $16-23^{\mathrm{b}}$ & 4D orthorhombic(incommensurately modulated) & $F m m m(00 \gamma) 0 s 0^{c}$ \\
\hline $\mathrm{V}$ & $23^{\mathrm{b}}-29$ & 5D tetragonal (incommensurately modulated) & $I 4 / m m m(\alpha \alpha 0) 000 s(-\alpha \alpha 0) 0000$ \\
\hline II & $29-43$ & Orthorhombic & Immm $[8]$ \\
\hline III & $43-55$ & Tetragonal & $I 4 / \mathrm{mmm}[11]$ \\
\hline IV & $55-(276)$ & Cubic & $F m-3 m[12,20]$ \\
\hline
\end{tabular}

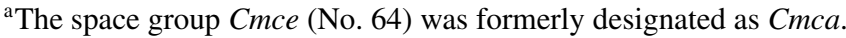

${ }^{\mathrm{b}}$ The transition pressure from VI to V was changed to $23 \mathrm{GPa}$ judging from the coexistence of their diffraction peaks at $23.2 \mathrm{GPa}$.

${ }^{\mathrm{c}}$ The $a$ and $c$ axes in the previous study [19] were swapped in this paper for easier comparison with the axes of the Cmce structure of phase I.

progressively deviated from those expected for the Cmce model in the pressure range from 16 to $21 \mathrm{GPa}$, we attributed the cause of this misfit to the strain induced by nonhydrostatic stress. Increased broadening of diffraction peaks further obscured the effect of a misfit of peak positions.

In our recent study of iodine with a helium pressuretransmitting medium [15], we again noticed the same problem for the patterns in this pressure range: the Rietveld fitting based on the Cmce model was not very successful for the diffraction patterns from 16 to $21 \mathrm{GPa}$. One of the possibilities could be that iodine transforms to another unknown structure $>16 \mathrm{GPa}$.

The first aim of this paper is to reanalyze the $\mathrm{x}$-ray diffraction data of iodine between 15 and $22 \mathrm{GPa}$ and search for a structural model that can successfully account for the misfit of peak positions. The second aim is to examine the crystal structures of phases V and VI by density functional theory (DFT) calculations.

\section{METHODS}

We used the same diffraction data of iodine as that used in our previous study $[15,19]$. In brief, iodine powder was pressurized with a helium pressure-transmitting medium in a diamond-anvil cell (DAC) [21]. Pressure was determined with the ruby luminescence method [22]. Powder X-ray diffraction experiments were done on beamline BL-13A (former beamline) of the Photon Factory, KEK. The beams were monochromatized to a wavelength of $0.42620(4) \AA$ and collimated to a beam size of $50 \mu \mathrm{m} \phi$. Diffraction patterns were taken by an imaging plate detector with a typical exposure time of $5 \mathrm{~min}$. A diamond-backing plate was used as a window in the DAC to collect full Debye-Scherrer rings [23], which were converted to $1 \mathrm{D} 2 \theta$-intensity patterns by the software PIP [24]. All the experiments were done at room temperature. We used JANA2006 [25] for the analysis of the incommensurately modulated structure and BIOVIA Materials Studio (MS) Reflex [26] for the analysis of the conventional structures. The March-Dollase-type preferred orientation corrections [27] were employed in the Rietveld analyses.

DFT calculations were done with MS CASTEP [28] with the generalized gradient approximation Perdew-BurkeErnzerhof functionals [29] and norm-conserving pseudopotentials [30,31]. For molecular dynamics (MD) calculations, we used the thermostat by Nosé [32] and a barostat in the Parrinello-Rahman scheme [33]. The time step was $5 \mathrm{fs}$, and the simulation time was 10 ps (2001 points).

\section{STRUCTURE ANALYSIS}

\section{A. Phase VI}

The diffraction patterns up to $14.6 \mathrm{GPa}$ fit well with the Cmce model of phase I, while the patterns from 16.9 to 21.8 GPa had misfit problems. Figure 1 shows the diffraction pattern obtained at $21.8 \mathrm{GPa}$ and the fit with the Cmce model by the Rietveld method. The lattice parameters converged to $a=5.748 \AA, b=3.933 \AA$, and $c=9.032 \AA$. In Fig. 1, the observed and calculated peak positions agree well for the peaks like 200, 112, 404, and 208, which have an even number $l$ of the Miller indices $h k l$, as displayed by the black peak bars and indices. On the other hand, the calculated positions for the peaks with an odd number $l$ (e.g., 111, 113, 133, 135, and 227), as displayed by the red peak bars and indices, show a significant deviation from the observed ones. Figure 1(b) shows similar peak shifts for many of the odd $l$ peaks in the higher angle region. In addition, the background optimized by the Rietveld analysis converges to a higher level than the experimental one. This is because the Cmce model does not fit 

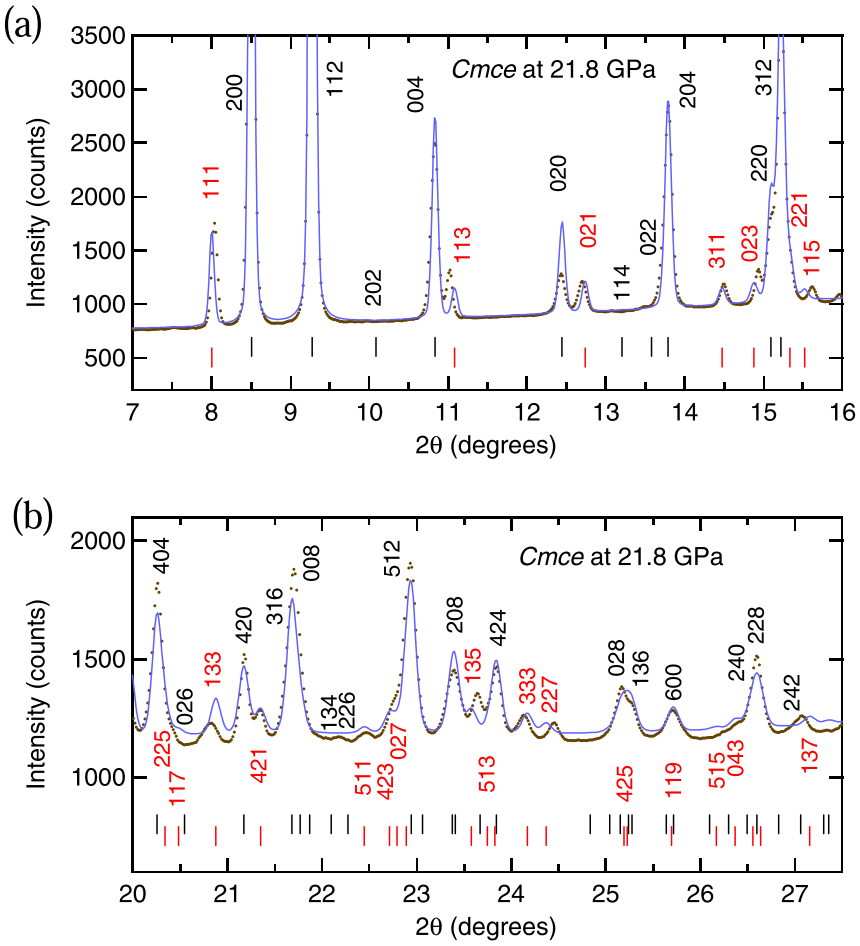

FIG. 1. Analysis with the previous three-dimensional Cmce model. The powder diffraction pattern of iodine observed at $21.8 \mathrm{GPa}$ and room temperature (black dots) and its Rietveld fit with the Cmce model (blue line) in (a) low- and (b) high-angle regions. The intensity scale is increased to make it easier to see the fit. The reliability factor for the fit was $R_{\mathrm{wp}}=5.37 \%$. The black vertical bars at the bottom show the positions of peaks having Miller indices $h k l$ with even $l$. The red vertical bars show the peaks with odd $l$. Indices are shown only for major reflections.

the peak positions, intensities, and overall profile. If we fix the background to the experimental level, $R_{\mathrm{wp}}$ increases, and the fitting becomes even worse. We thus conclude that the crystal structure of iodine in this pressure range is different from the Cmce model. This implies that there is an intermediate phase, which we call phase VI.

We tried fitting the pattern with a monoclinic or a triclinic cell distorted from the orthorhombic Cmce structure, including the monoclinic $C 2 / m$ model proposed by Zeng et al. [34]. However, no successful results were obtained (see Fig. S1 in the Supplemental Material [35]). Generally, there is a oneto-one correspondence between a structure and a diffraction pattern, except for enantiomers. However, the accuracy and angular restrictions of the diffraction pattern may yield multiple structural models. It is necessary to verify whether the interatomic distances and bond angles are normal and to narrow down the candidates. One method to avoid uncertainty in the model is to take advantage of the space group constraints to reduce the number of structural parameters as much as possible. Another method is to evaluate enthalpies, atomic coordinates, and lattice stability using theoretical calculations, which are indispensable for recent structural analysis. We have adopted both methods in our structural analyses. Thus, we considered the possibility that an incommensurate modulation occurs in phase VI in a similar way to phase V. We
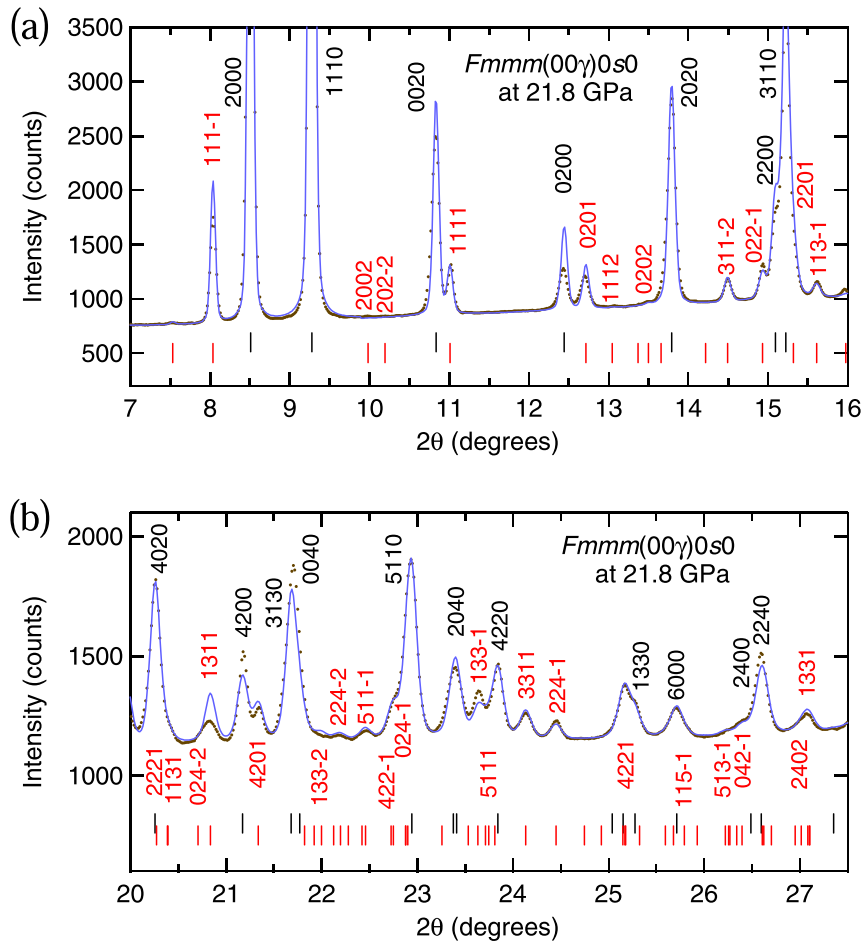

FIG. 2. Analysis with the present four-dimensional (4D) model. Rietveld analysis of the pattern at $21.8 \mathrm{GPa}$ with the $\mathrm{Fmmm}(00 \gamma) 0 \mathrm{~s} 0$ model in (a) low- and (b) high-angle regions. The intensity scale is increased to make it easier to see the fit. The reliability factor was $R_{\mathrm{wp}}=4.13 \%$. The black vertical bars at the bottom show fundamental $m=0$ reflections with 4D Miller indices $h k l m$. The red vertical bars show satellite $m \neq 0$ peaks. Note that both the positive and negative $m$ peaks are allowed to appear. Indices are shown only for major reflections.

made an orthorhombic unit cell (space group Fmmm) that has the $c$ axis equal to half the length of the $c$ axis of the Cmce unit cell. Then we introduced a modulation along the $c$ axis with a modulation wave number $\gamma$ slightly different from $\frac{1}{2}$. Figures 2(a) and 2(b) show the result of the Rietveld fitting in the lower and higher $2 \theta$ regions, respectively. The observed peak positions fit much better than those shown in Figs. 1(a) and 1(b). The misfit of the background in Fig. 1(b) is completely resolved. This is because the peak profile created by the model is in excellent agreement with the experiment. This structural model for phase VI is described by four-dimensional (4D), or more precisely, $(3+1)$-dimensional superspace group Fmmm $(00 \gamma) 0 s 0$ (No. 69.1.17.2 [36] or No. 455 [37]), which is the same as for phase V [19]. The basic structure is Fmmm [face-centered orthorhombic (fco)], where the atoms occupy the special position $(0,0$, $0)$. This model has five refinable structural parameters: four lattice parameters $a, b, c$, and modulation wave number $\gamma$, and one modulation amplitude $y \sin 1$. They converged to $a=5.7483(6) \AA, b=3.9339(4) \AA, c=4.5157(5) \AA, \gamma=$ $0.4817(5)$, and $y \sin 1=0.0752(8)$ at $21.8 \mathrm{GPa}$. The reliability factor was $R_{\mathrm{wp}}=4.13 \%$. In comparison, the previous Cmce model gave $R_{\mathrm{wp}}=5.37 \%$ with the same five refinable parameters: three lattice parameters $a, b, c$, and two atomic coordination parameters $y$ and $z$. In the measured diffraction 


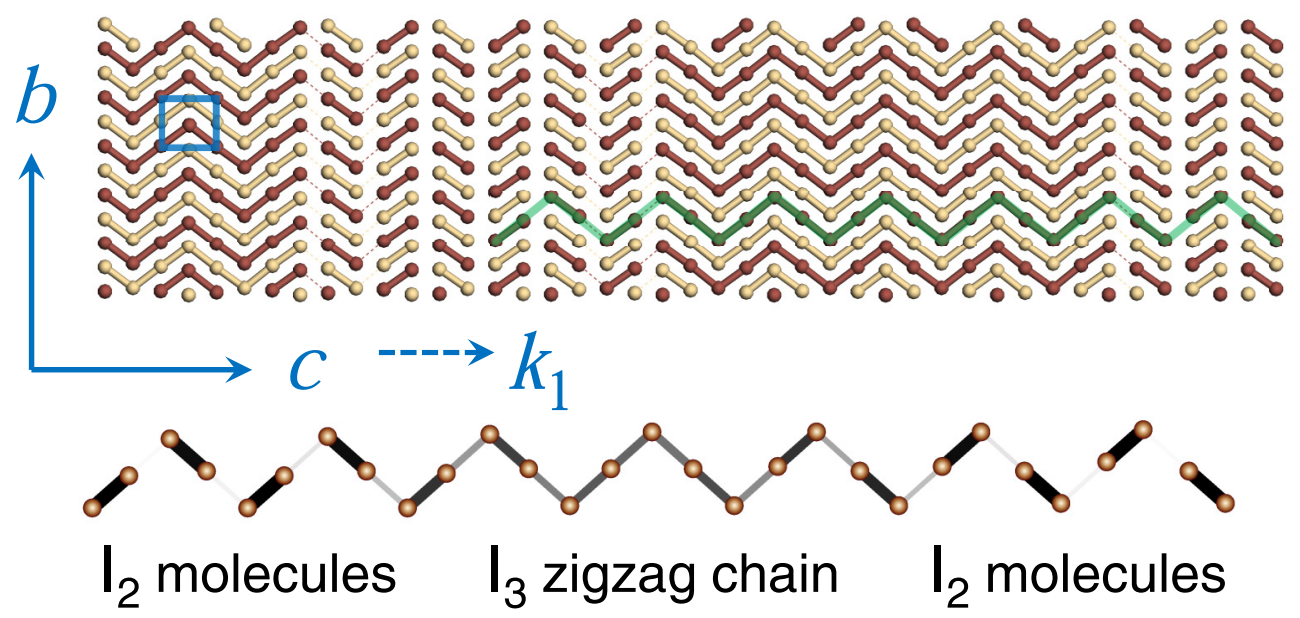

FIG. 3. Four-dimensional incommensurate structure of phase VI at $21.8 \mathrm{GPa}$. The blue rectangle and solid arrows show the basic lattice and its crystalline axes, respectively. The blue dashed arrow shows the modulation direction $k_{1}$ that corresponds to the fourth axis. The figure contains $1 \times 5 \times 20$ cells of the basic lattice. Brown and yellow atoms are in the plane at $x=0$ and $\frac{1}{2}$, respectively. Thick bonds connect atoms at distances $<2.90 \AA$, and thin broken lines connect those in the range 2.90-2.95. . The overall nearest interatomic distances are distributed from 2.75-3.28 $\AA$. The bottom figure shows the arrangement of atoms along the green line in the top figure. It illustrates how the interatomic distances vary within an $\mathrm{I}_{3}$ zigzag chain. Thicker and darker-colored bonds show shorter distances. One can see that the nearest neighbor distances are almost equal in the middle of the zigzag chain, while short distances periodically appear at both ends and finally form $\mathrm{I}_{2}$ molecules.

pattern, 57 peaks are clearly identified. The fact that these diffraction intensities can be explained by a single atomic coordinate parameter $y \sin 1$ guarantees the uniqueness of this structure.

Figure 3 shows the finally obtained 4D structure. Two different regions appeared alternately along the $c$ axis: one with a Cmce-like arrangement of $\mathrm{I}_{2}$ molecules and another with zigzag chains of three iodine atoms. The two regions are continuously connected than clearly separated, as shown in the lower part of Fig. 3. The patterns at 16.9 and 19.1 $\mathrm{GPa}$ were also successfully analyzed with this model, as shown in Table S1 in the Supplemental Material [35]. The Rietveld fit of the pattern at $14.6 \mathrm{GPa}$, on the other hand, gave $\gamma=0.5003(5)$, which is indistinguishable from $\frac{1}{2}$ within the fitting errors. We therefore conclude that iodine at $14.6 \mathrm{GPa}$ is still in phase I. The modulation wave number $\gamma$ decreases progressively from $\frac{1}{2}$ at $14.6 \mathrm{GPa}$ to 0.482 at $21.8 \mathrm{GPa}$ (Table S1 in the Supplemental Material [35]). The decrease of $\gamma$ seems to be continuous from $\frac{1}{2}$, suggesting that the transition from phase I to VI could be of the second order. On the other hand, phases VI and V coexist at $23.2 \mathrm{GPa}$, and there are discernible gaps in their lattice parameters and volumes. These facts clearly indicate that the transition between phases VI and V is of the first order. The crystal structures of bromine [38] and chlorine [39] around their molecular dissociation pressures were recently analyzed with the $C 2 / \mathrm{m}$ model of Zeng et al. [34]. It would be worth analyzing them also with our 4D model of phase VI of iodine.

In the previous structural model for phase $\mathrm{V}$ [15], the modulation wave number $\alpha$ took a value close to $\frac{1}{4}$. This poses a question as to whether the first-order transition from phase VI to $\mathrm{V}$ only halves the modulation wave number from $\sim \frac{1}{2}$ to $\frac{1}{4}$. To try and answer this, we simulated the VI-to-V transition and the structures of each phase by MD with the use of DFT calculations. We constructed a $2 \times 4 \times 2$ supercell of the Cmce structure of phase I at $16 \mathrm{GPa}$ including 128 atoms and used it as an initial structure for the MD simulation. The MD simulation was done in an isothermal-isobaric (NPT) ensemble at a pressure of $16 \mathrm{GPa}$ and a temperature of $150 \mathrm{~K}$. Figure 4 shows the final frame of the simulation. The zigzag

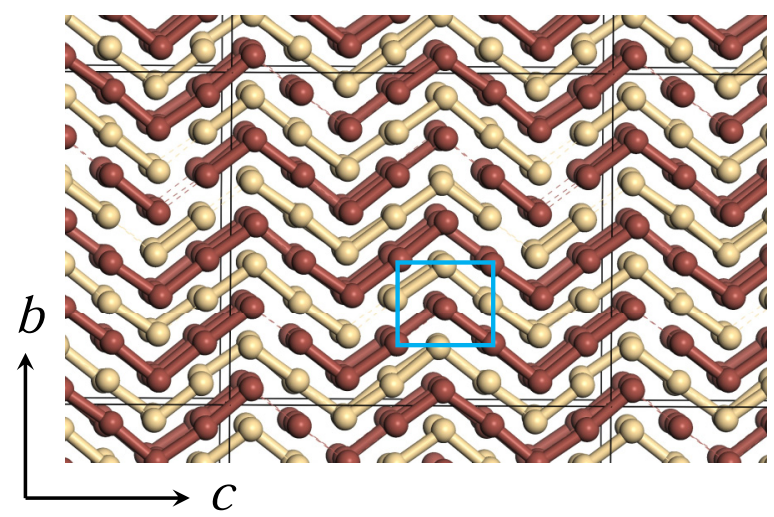

FIG. 4. Final frame of phase VI after molecular dynamics (MD) simulation at $16 \mathrm{GPa}$ and $150 \mathrm{~K}$. As an initial structure, a $2 \times 4 \times 2$ supercell of the Cmce model of phase I at $16 \mathrm{GPa}$ with 128 atoms was used. The MD simulation was done in an isothermal-isobaric (NPT) ensemble. The simulation pressure is in the region where phase VI is stable. The black lines and arrows show the periodic boundaries and the axes of the simulation cell, respectively. To show the atoms along the $a$ axis, the $a$ axis is tilted slightly from perpendicular to the screen. Brown and yellow atoms are in the plane at $x=0$ and $\frac{1}{2}$, respectively. Thick lines indicate interatomic distances (bonds) $<2.90 \AA$, and thin broken lines show those in the range 2.90-2.95 $\AA$. We obtained a four-dimensional (4D) modulated structure, which has been hitherto assigned to phase $\mathrm{V}$, but with a different modulation wave number. The blue rectangle represents the basic lattice of the 4D structure. 


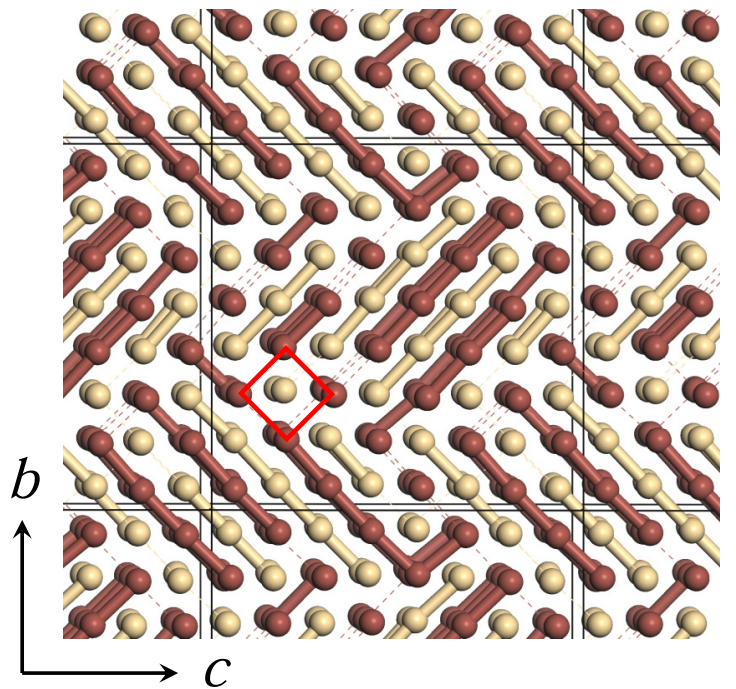

FIG. 5. Final frame of phase $\mathrm{V}$ after molecular dynamics (MD) at $24 \mathrm{GPa}$ and $150 \mathrm{~K}$. A $2 \times 4 \times 2$ supercell of the Cmce structure with 128 atoms was used for the MD. The MD simulation was done in an isothermal-isobaric (NPT) ensemble. The simulation pressure is in the region where phase $\mathrm{V}$ is stable. The black lines and arrows show the periodic boundaries and the axes of the simulation cell, respectively. To show the atoms along the $a$ axis, the $a$ axis is tilted slightly from perpendicular to the screen. Brown and yellow atoms are located in the plane at $x=0$ and $\frac{1}{2}$, respectively. Thick lines indicate interatomic distances (bonds) $<2.90 \AA$, and thin broken lines show those in the range 2.90-2.95 $\AA$. We obtained a tetragonallike structure. The red square represents the basic lattice of the fivedimensional structure.

chains of atoms running from left to right in the direction of the $c$ axis can be clearly seen. The thick and broken lines moved dynamically with time. This means that the covalent bonds of adjacent $\mathrm{I}_{2}$ molecules like I-I ...I-I are temporarily lost, and new bonds of different pairs of iodine atoms form like I ...I-I ...I. Nevertheless, the same chains always stacked in the direction of the $b$ axis, and no chains were formed in this direction. Throughout the whole process of the simulation, we observed that the $c$ axis remains larger than the $b$ axis, the chains always run in the direction of the $c$ axis, and the atomic displacement occurs in the direction of the $b$ axis. This is the cause of the partial break of $\mathrm{I}_{2}$ covalent bonds and the formation of new ones. The dynamical picture of the crystal structure of phase VI thus obtained matches well to the MD simulation.

\section{B. Phase V}

We have also performed a similar MD simulation for iodine at $24 \mathrm{GPa}$ starting from the initial structure of a $2 \times 4 \times 2$ supercell of the Cmce structure. Figure 5 shows the final frame of the simulation. The thick lines (bonds) and broken lines moved dynamically with time. In this simulation, the lengths of the $b$ and $c$ axes became nearly equal, and the chains broke into small pieces. Two types of bonds appeared, pointing toward the upper left or upper right. No chains appeared, in contrast to the case shown in Fig. 4. There are two different regions, where $\mathrm{I}_{2}, \mathrm{I}_{3}$, or $\mathrm{I}_{4}$ units align in the upper

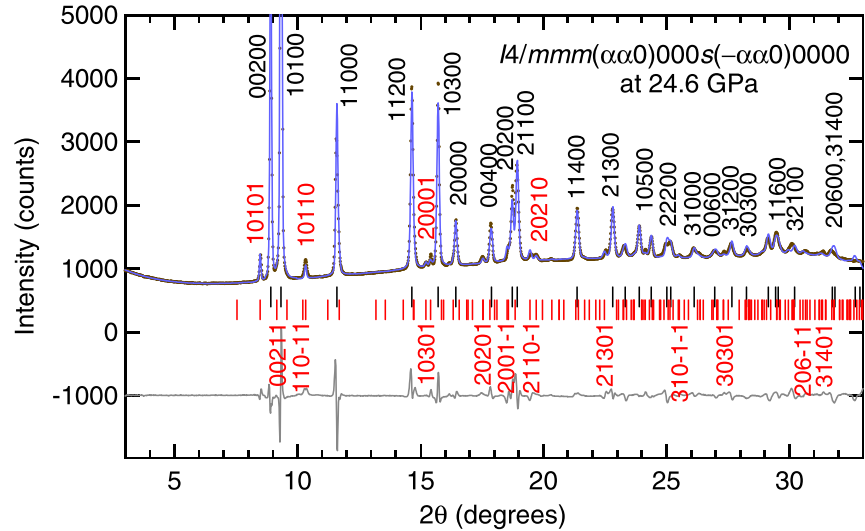

FIG. 6. Analysis with the five-dimensional (5D) model. Rietveld analysis of the pattern of iodine phase $\mathrm{V}$ at $24.6 \mathrm{GPa}$ and at room temperature with the $5 \mathrm{D}$ superspace group. The intensity scale is increased to make it easier to see the fit. The reliability factor was $R_{\text {wp }}=4.07 \%$. The red vertical bars show $m \neq 0$ or $n \neq 0$ satellite reflections. Miller indices hklmn are shown only for major reflections.

left or upper right directions. This structure contradicts the incommensurately modulated 4D structure $F m m m(\alpha 00) 0 s 0$ previously proposed for phase V [19]. This poses a question as to which one is the correct structure for phase V. The structure shown in Fig. 5 can be described by a tetragonal cell, as displayed by the red square, having modulations in two directions. The corresponding superspace group is five dimensional (5D), or more precisely, $(3+2)$ dimensional I4/mmm $(\alpha \alpha 0) 000 s(-\alpha \alpha 0) 0000 \quad$ (No. 139.2.70.12) [36], which is equivalent to $I 4 / m m m(\alpha \alpha 0, \alpha-\alpha 0) 00 m g$ (No. 3150) [37].

Figure 6 shows the result of the Rietveld fitting with this superspace group. The basic structure of this model is $14 / \mathrm{mmm}$ [body-centered tetragonal (bct)], where the atoms occupy the special position $(0,0,0)$. There are four modulation amplitude parameters: $x \sin 1, y \sin 1, x \sin 2$, and $y \sin 2$. They are bound by the symmetry constraint as $x \sin 1=-y \sin 1=x \sin 2=y \sin 2$. Hence, the 5D model has four refinable structural parameters: three lattice parameters $a, c$, and modulation wave number $\alpha$, and one modulation amplitude $x \sin 1$. The optimization of these four structural parameters successfully explains the 42 peaks that are clearly identified in the measured diffraction patterns. Using the Rietveld analysis with this superspace group, the structural parameters converged to $a=2.9815(3) \AA, c=5.4849(6) \AA, \alpha=0.1289(5)$, and $x \sin 1=0.0341(5)$ at $24.6 \mathrm{GPa}$. The reliability factor was $R_{\mathrm{wp}}=4.07 \%$. This is reasonably small in view of the fact that the present model uses one less refinable parameter than the previous 4D Fmmm $(\alpha 00) 0 s 0$ model, which used five structural parameters: $a, b, c$, modulation wave number $\mathbf{k}$, and modulation amplitude $B_{1}(y)$ [19]. This means that the reliability of the structural model has increased.

The resulting structure is shown in Fig. 7 and agrees well with the MD result in Fig. 5. Because of the tetragonal symmetry of the basic lattice, two modulation waves simultaneously propagate in the orthogonal directions $\mathbf{a}+\mathbf{b}$ and $-\mathbf{a}+\mathbf{b}$. One can see that the population of $\mathrm{I}_{2}$ is much smaller than that in phase VI, shown in Fig. 3. Distance distribution 


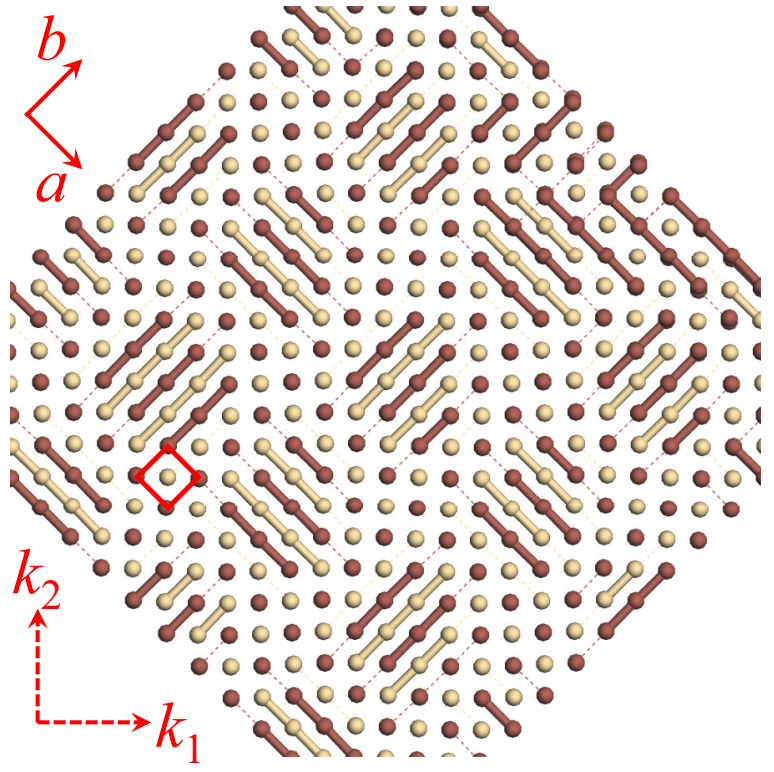

FIG. 7. Five-dimensional incommensurate structure of phase $\mathrm{V}$ at 24.6 GPa. The red square and solid arrows show the basic lattice and its crystalline axes, respectively. The red dashed arrows show modulation directions $k_{1}$ and $k_{2}$ that correspond to the fourth and fifth axes, respectively. The figure contains $15 \times 15 \times 1$ cells of the basic lattice. Brown and yellow atoms are in the plane at $z=0$ and $\frac{1}{2}$, respectively. Thick bonds connect atoms at distances $<2.90 \AA$, and thin broken lines connect those in the range $2.90-2.95 \AA$. The overall nearest interatomic distances are distributed from 2.79 to $3.17 \AA$.

of phase V from 2.79 to $3.17 \AA$ shows a broad peak at $3.0 \AA$. This means that the molecular dissociation proceeds further in phase V. This paper, which combines DFT calculations with the Rietveld analysis, clearly shows that the 5D tetragonal superspace group is more plausible than the $4 \mathrm{D}$ orthorhombic one. It should be noted that, in our previous study [15], we also proposed a 5D superspace group $\operatorname{I} 4 m m(-\alpha \alpha 0, \alpha \alpha 0) 0 g g$ (No. 2794) [37] as an alternative structure for phase V, which has lower symmetry than the present one. The superspace group at that time was noncentrosymmetric, and there were extra atomic parameters. We made a thorough analysis of superspace groups and found that the structure of phase $\mathrm{V}$ can be represented by a centrosymmetric superspace group with higher symmetry. We believe that the present superspace group is crystallographically more appropriate. The lower side of Table I summarizes the structural evolution of iodine obtained by this paper.

\section{DISCUSSION}

The most important finding of this paper is that the first stage of the pressure-induced molecular dissociation of iodine starts in its molecular state. The structure of phase VI can be characterized as a mixture of two regions: one having $\mathrm{I}_{2}$ molecules as in phase I and another having zigzag chains of three iodine atoms. The two regions cannot be clearly separated; however, they are connected continuously and appear repeatedly with a modulation wave propagating along the $c$ axis. The zigzag chains of three iodine atoms imply the partial break of the covalent bond of $\mathrm{I}_{2}$ diatomic molecules and for- mation of new ones. The second stage is the VI-V transition in which modulation of atomic positions extends into $2 \mathrm{D}$ in the molecular plane. The intermediate phases between molecular and nonmolecular structures could be difficult to represent with a normal crystal structure. Therefore, iodine might have taken a way to use incommensurability to accommodate both molecular and nonmolecular units into a single modulated structure of phase VI. The transition to phase $\mathrm{V}$ is a $2 \mathrm{D}$ version of this incommensurability.

The persistence of the molecular nature in phase VI is in accordance with the previous results of Mössbauer spectroscopy and Raman-scattering experiments. Pasternak et al. [17] have conducted a high-pressure Mössbauer spectroscopy experiment on iodine and reported that phases HP1 and HP2 appeared $>16$ and $24 \mathrm{GPa}$, respectively. They proposed a structural model for phase HP1 composed of zigzag chains of $\mathrm{I}_{2}$ molecules (or two iodine atoms) extending along the $b$ axis. It should be noted that the same structure with zigzag chains was also proposed theoretically by considering the distortions in the bond occupation probabilities [40]. This structure, however, assumes the same space group as phase I (Cmce). We have already shown that this space group cannot account for the x-ray diffraction pattern observed in this pressure range. If the present structural model for phase VI, which contains zigzag chains of three atoms extending along the $c$ axis, can explain the observed Mössbauer spectra for phase HP1, then the two experimental observations would become consistent. Furthermore, the phase HP2, which was proposed to have a 2D network of iodine atoms, would conform to phase $\mathrm{V}$ that is now shown to have incommensurate modulations in two directions. We can summarize the overall structural change of iodine as follows: from the diatomic molecular structure (phase I) to the 1D chain structure (phase VI or HP1) and to the 2D network structure (phase V or HP2). In the previous studies, the pressure for the molecular dissociation of iodine was set at 21-24 GPa, where phase I disappears. However, due to the discovery of a transition sequence and its comparison with the Mössbauer experiment, it became necessary to distinguish between the starting pressure $P_{s}$ and the finishing pressure $P_{f}$ of molecular dissociation. Based on the present structural analysis, $P_{s}$ is given as $16 \mathrm{GPa}$, where phase VI appears, and $P_{f}$ as $29 \mathrm{GPa}$, where phase $\mathrm{V}$ disappears.

Two independent Raman-scattering measurements were performed on iodine under high pressure $[16,18]$. These experiments showed bands called $X$ and $Y$ that appeared under pressures $>10-15 \mathrm{GPa}$, while keeping the stretching $\left[A_{g}^{(\mathrm{S})}\right.$ and $\left.B_{3 g}^{(\mathrm{S})}\right]$ and librational $\left[A_{g}^{(\mathrm{L})}\right.$ and $\left.B_{3 g}^{(\mathrm{L})}\right]$ modes intact. Although the appearing pressures of the $X$ and $Y$ bands are different from the I-VI transition pressure determined in the present $\mathrm{x}$-ray analysis, it is reasonable to assume that the $X$ and $Y$ bands are intimately related to phase VI. The persistence of the $A_{g}^{(\mathrm{S})}$ and $B_{3 g}^{(\mathrm{S})}$ stretching modes implies that $\mathrm{I}_{2}$ molecular units still exist in phase VI. As shown in Fig. 3, the structure of phase VI consists of two characteristic regions, one dominated by $I_{2}$ molecules and another by zigzag chains made up of three iodine atoms. We can easily imagine that the former region is responsible for the Raman bands characteristic of $\mathrm{I}_{2}$ molecules. We thus suppose that the $X$ and $Y$ bands originate from the zigzag chains. We calculated the frequencies 
(a)

(c)
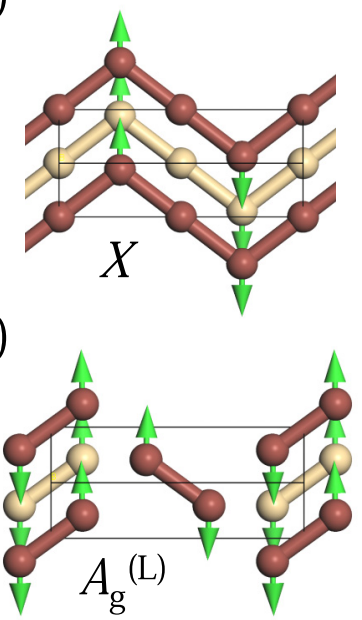

(b)

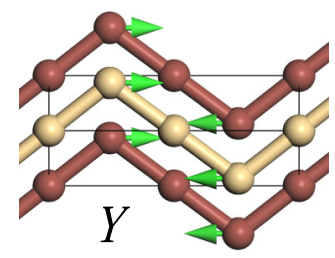

(d)

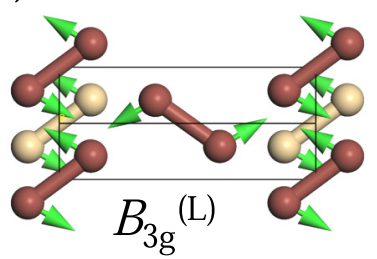

(e)

\begin{tabular}{|c|c|c|}
\hline Raman band & $\begin{array}{c}\text { Experiment [16] } \\
\text { Wavenumber }\left(\mathrm{cm}^{-1}\right)\end{array}$ & $\begin{array}{c}\text { Calculation } \\
\text { Wavenumber }\left(\mathrm{cm}^{-1}\right)\end{array}$ \\
\hline$X$ & 100 & 84.0 \\
\hline$A_{\mathrm{g}}{ }^{(\mathrm{L})}$ & 105 & 87.8 \\
\hline$B_{3 \mathrm{~g}}{ }^{(\mathrm{L})}$ & 135 & 130.4 \\
\hline$Y$ & 141 & 139.6 \\
\hline
\end{tabular}

FIG. 8. Representative Raman active modes for two local structures in iodine phase VI obtained by density functional theory (DFT) calculations at $14 \mathrm{GPa}$. (a) $X$ and (b) $Y$ bands for the $C m c m$ zigzag chain region. (c) $A_{g}^{(\mathrm{L})}$ and (d) $B_{g}^{(\mathrm{L})}$ bands for the Cmce molecular region. (e) Comparison of the experimental and calculated Raman frequencies.

of possible Raman modes for this region by approximating the local structure with a $\mathrm{Cmcm}$ cell. There are three Ramanactive modes, of which two are relatively strong, as shown in Figs. 8(a) and 8(b). The former is vibration perpendicular to the chain direction, and the latter is along the chain direction. For comparison, we also calculated the Raman frequencies for the $\mathrm{I}_{2}$ region by approximating its structure with a Cmce cell, as shown in Figs. 8(c) and 8(d). The result is compared with the experimental data of the study by Kume et al. [16] in Fig. 8(e). The agreement is quite satisfactory. Our calculations further indicate that the $X$ band shows softening with pressure, while the $Y$ band shows hardening, which agrees with the experiment.

The phase transition to an incommensurate structure near the band gap closure implies that the transition is related to the electronic band structure. Duan et al. [41] and San et al. [42] performed DFT calculations of elastic constants and band structures for bromine and iodine, respectively. They found that the elastic constant $C_{44}$ of the Cmce model and the unmodulated fco model softens and modulated structures appear. These papers, however, used a structure model with eight atoms lined up in the $c$-axis direction. We attempted to verify the phase transition by DFT calculations using a long-period model that more closely approximates the experimentally observed incommensurate structure. We multiplied the basic fco structure of phase VI by 15 to create a model with

30 iodine atoms aligned in the $c$-axis direction. The structure was optimized at $18 \mathrm{GPa}$. As can be seen in Fig. S2 in the Supplemental Material [35], $\mathrm{I}_{2}, \mathrm{I}_{3}$, and short chains appeared sporadically. The enthalpy per one atom in this case was $4.0 \mathrm{meV}$ lower than the Cmce model. This suggests that the electronic structure would be responsible for the appearance of the incommensurate phase VI. The present finding of phase $\mathrm{VI}>16 \mathrm{GPa}$ poses a question as to whether iodine becomes metallic in phase I or VI. Our energy band calculation for the Cmce model within the DFT scheme shows that the energy gap closes at 18.0 GPa. The same calculation with the longperiod model including 30 atoms resulted in a metallic state even at a lower pressure of $10 \mathrm{GPa}$. This seems to indicate that phase I remains an insulator up to the transition pressure at $16 \mathrm{GPa}$, and the metallization first occurs in phase VI. The origin of the incommensurate phase III of tellurium was explained by the combined effects of electron-phonon coupling and Fermi surface nesting [43]. A similar detailed analysis of the band structure of iodine is desirable to clarify the origin of incommensurate modulation and possible concurrence of metallization and structural phase transition. We do not evaluate the metallization pressure in more detail here because there is a general tendency to underestimate the band gap in DFT calculations and because larger supercells are needed for accurate band calculations of incommensurate structures.

As shown in Figs. 3 and 7, phase VI has $\mathrm{I}_{3}$ zigzag chains, and phase $\mathrm{V}$ contains $\mathrm{I}_{3}$ and $\mathrm{I}_{4}$ units. These local structures are known as polyiodide ions [44]. The triiodide $\left(\mathrm{I}_{3}^{-}\right)$and tetraiodide $\left(\mathrm{I}_{4}^{2-}\right)$ ions are linear and found in some iodine compounds [45]. The pentaiodide ion $\left(\mathrm{I}_{5}^{-}\right)$, on the other hand, has a bent structure. Longer polyiodide ions with chain structures are also known [46]. Theoretical and computational studies of the structure of iodine on graphene sheets also show that the straight $I_{3}$ and bent $I_{5}$ are stable [47]. These references indicate that the ionization and reconstruction of $\mathrm{I}_{2}$ molecules are closely related. It follows that the chain part of phase VI and the $I_{3}$ and $I_{4}$ parts of phase $V$ could also appear for electronic reasons, and the entire crystal is expected to undergo metallization.

The superconducting state $>28 \mathrm{GPa}$ found by Shimizu et al. $[13,14]$ was considered to occur in the monatomic phase II after completing molecular dissociation. The pressure of 28 $\mathrm{GPa}$ is in the $5 \mathrm{D}$ phase $\mathrm{V}$ according to our phase diagram (lower side of Table I) but is close to the boundary between phase V and II. It would be interesting to clarify which phase first shows superconductivity and whether the incommensurate modulation would relate to superconductivity.

Among the elements, only Eu is known to show a pressureinduced incommensurate-to-incommensurate phase transition (Eu-IV to Eu-V at $38 \mathrm{GPa}$ ) between modulated structures, excluding the phase transitions between host-guest structures [48]. This transition accompanies a discontinuous jump in the modulation wavelength and amplitude, while keeping the same monoclinic 4D superspace group. The VI-V phase transition of iodine in this paper is also an incommensurate-toincommensurate one between modulated structures, but this is the first case in which the transition accompanies a change in the dimension of the superspace group from 4D to 5D.

Theoretical studies on the molecular dissociation of other halogens have been conducted recently. Li et al. [49] 
explored the stability of long-period structures for bromine. They predicted the existence of four successive phases (oF20, oC24, oF28, oC12) between the molecular phase I (Cmce) and the monatomic phase II (bco, Immm). Interestingly, the oF28 seems to be close to our incommensurate structure of phase VI. Their calculations have yet to find a model that resembles phase V. Duan et al. [50] performed theoretical calculations of successive phase transitions of fluorine leading to the molecular dissociation. They proposed a $P 6 / m c c$ structure at $2.7 \mathrm{TPa}$, which consists of $\mathrm{F}_{2}$ molecules and $\mathrm{F}$ chains, a $P m-3 n$ structure at $4 \mathrm{TPa}$ having $\mathrm{F}$ atoms and $\mathrm{F}$ chains, and an Fddd structure at $30 \mathrm{TPa}$, which is completely monatomic. Although long-period modulated structures have not been reported for fluorine, multistep transitions seem to be common to molecular dissociations of many molecular crystals.

Dense hydrogen, which is also expected to show metallization and molecular dissociation at ultrahigh pressures [51,52], is theoretically predicted to become a room-temperature superconductor [53,54]. A recent quantum Monte Carlo simulation has predicted that the atomic hydrogen would appear at $375 \mathrm{GPa}$ [55]. Further, a very recent x-ray diffraction study with a DFT calculation showed that the solid hydrogen was still in molecular state at $300 \mathrm{GPa}$ [56]. Between these pressures, modulated phases of solid hydrogen might emerge.

\section{CONCLUSIONS}

The molecular dissociation of iodine is now clarified to proceed in three stages. The I-VI phase transition is the first one, in which the I-I covalent bonds partially break and zigzag chains of three iodine atoms are constructed. However,
$\mathrm{I}_{2}$ molecules still exist in some regions, indicating a mixed molecular and nonmolecular nature of phase VI. The structure of phase VI is consistent with the pictures obtained in a previous Mössbauer experiment as well as in Raman-scattering experiments. The second stage is the VI-V transition. The population of $\mathrm{I}_{2}$ molecule becomes much smaller than that of phase VI. Since one can still see linear chains of three or four iodine atoms, the structure cannot be said to be monatomic. The third stage is the V-II transition, which signifies complete molecular dissociation. The incommensurate phases VI and V are quite interesting from the viewpoint of intermediate states between molecular and nonmolecular ones. To the best of our knowledge, this is the first pressure-induced phase transition in which the superspace group changes from 4D to 5D ones. We wait for further theoretical investigations on the mechanism of phase transitions and change of physical properties. There are many intriguing questions about pressure-induced metallization and molecular dissociation of molecular solids. For example, it will be very interesting to investigate how the regions of phases VI and V change at high and low temperatures. We hope that this paper will stimulate further experimental and theoretical work including those on other solid halogens [37,38,57-60].

\section{ACKNOWLEDGMENTS}

The original powder x-ray diffraction data were taken at the Photon Factory, KEK, under Proposal No. 2001G225. We would like to thank Dr. Kyoko Sato for obtaining diffraction data in 2001. We also would like to thank Prof. Yasuhiko Fujii and Prof. Osamu Shimomura for their encouragement.
[1] A. S. Balchan and H. G. Drickamer, Effect of pressure on the resistance of iodine and selenium, J. Chem. Phys. 34, 1948 (1961).

[2] B. M. Riggleman and H. G. Drickamer, Temperature coefficient of resistance of iodine and selenium at high pressure, J. Chem. Phys. 37, 446 (1962).

[3] B. M. Riggleman and H. G. Drickamer, Approach to the metallic state as obtained from optical and electrical measurements, J. Chem. Phys. 38, 2721 (1963).

[4] O. Shimomura, K. Takemura, Y. Fujii, S. Minomura, M. Mori, Y. Noda, and Y. Yamada, Structure analysis of high-pressure metallic state of iodine, Phys. Rev. B 18, 715 (1978).

[5] F. van Bolhuis, P. B. Koster, and T. Migchelsen, Refinement of the crystal structure of iodine at $110^{\circ} \mathrm{K}$, Acta Cryst. 23, 90 (1967).

[6] The space group Cmce (No. 64) was formerly designated as Cmca.

[7] K. Takemura, Y. Fujii, S. Minomura, and O. Shimomura, Pressure-induced structural phase transition of iodine, Solid State Commun. 30, 137 (1979).

[8] K. Takemura, S. Minomura, O. Shimomura, and Y. Fujii, Observation of Molecular Dissociation of Iodine at High Pressure by X-Ray Diffraction, Phys. Rev. Lett. 45, 1881 (1980).

[9] K. Takemura, S. Minomura, O. Shimomura, Y. Fujii, and J. D. Axe, Structural aspects of solid iodine associated with metallization and molecular dissociation under high pressure, Phys. Rev. B 26, 998 (1982).

[10] The molecular dissociation pressure of $21 \mathrm{GPa}$ in early x-ray diffraction studies [7-9] refers to the starting pressure of dissociation $P_{s}$. To avoid confusion, we made a clear distinction between the starting pressure $P_{s}$ and the finishing pressure $P_{f}$ in this paper.

[11] Y. Fujii, K. Hase, Y. Ohishi, N. Hamaya, and A. Onodera, Pressure-induced monatomic tetragonal phase of metallic iodine, Solid State Commun. 59, 85 (1986).

[12] Y. Fujii, K. Hase, N. Hamaya, Y. Ohishi, A. Onodera, O. Shimomura, and K. Takemura, Pressure-Induced Face-Centered-Cubic Phase of Monatomic Metallic Iodine, Phys. Rev. Lett. 58, 796 (1987).

[13] K. Shimizu, N. Tamitani, N. Takeshita, M. Ishizuka, K. Amaya, and S. Endo, Pressure-induced superconductivity of iodine, J. Phys. Soc. Jpn. 61, 3853 (1992).

[14] K. Shimizu, T. Yamauchi, N. Tamitani, N. Takeshita, M. Ishizuka, K. Amaya, and S. Endo, The pressure-induced superconductivity of iodine, J. Supercond. 7, 921 (1994).

[15] K. Takemura, K. Sato, H. Fujihisa, and M. Onoda, Modulated structure of solid iodine during its molecular dissociation under high pressure, Nature 423, 971 (2003).

[16] T. Kume, T. Hiraoka, Y. Ohya, S. Sasaki, and H. Shimizu, High Pressure Raman study of Bromine and Iodine: Soft Phonon 
in the Incommensurate Phase, Phys. Rev. Lett. 94, 065506 (2005).

[17] M. Pasternak, J. N. Farrell, and R. D. Taylor, Metallization and Structural Transformation of Iodine Under Pressure: A Microscopic View, Phys. Rev. Lett. 58, 575 (1987).

[18] H. Olijnyk, W. Li, and A. Wokaun, High-pressure studies of solid iodine by Raman spectroscopy, Phys. Rev. B 50, 712 (1994).

[19] K. Takemura, K. Sato, H. Fujihisa, and M. Onoda, Structural phase transitions in iodine under high pressure, Z. Krystallogr. 219, 749 (2004).

[20] R. Reichlin, A. K. McMahan, M. Ross, S. Martin, J. Hu, R. J. Hemley, H.-K. Mao, and Y. Wu, Optical, X-ray, and bandstructure studies of iodine at pressures of several megabars, Phys. Rev. B 49, 3725 (1994).

[21] K. Takemura, P. Ch. Sahu, Y. Kunii, and Y. Toma, Versatile gasloading system for diamond-anvil cells, Rev. Sci. Instrum. 72, 3873 (2001).

[22] C.-S. Zha, H.-K. Mao, and R. J. Hemley, Elasticity of MgO and a primary pressure scale to $55 \mathrm{GPa}$, Proc. Natl. Acad. Sci. USA 97, 13494 (2000).

[23] K. Takemura and S. Nakano, Performance of a synthetic diamond-backing plate for the diamond-anvil cell at ultrahigh pressures, Rev. Sci. Instrum. 74, 3017 (2003).

[24] H. Fujihisa, Intellectual property number H22PRO-1100 of AIST, Japan (2009).

[25] V. Petříček, M. Dušek, and L. Palatinus, Crystallographic computing system JANA2006: general features, Z. Kristallogr. 229, 345 (2014).

[26] Dassault Systèmes Americas Corp., BIOVIA Materials Studio Reflex Website, https://www.3ds.com/products-services/ biovia/products/molecular-modeling-simulation/bioviamaterials-studio/ (accessed 15 July 2021).

[27] W. A. Dollase, Correction of intensities for preferred orientation in powder diffractometry: application of the March model, J. Appl. Cryst. 19, 267 (1986).

[28] S. J. Clark, M. D. Segall, C. J. Pickard, P. J. Hasnip, M. I. J. Probert, K. Refson, and M. C. Payne, First principles methods using CASTEP, Z. Kristallogr. 220, 567 (2005).

[29] J. P. Perdew, K. Burke, and M. Ernzerhof, Generalized Gradient Approximation Made Simple, Phys. Rev. Lett. 77, 3865 (1996).

[30] N. Troullier and J. L. Martins, Efficient pseudopotentials for plane-wave calculations, Phys. Rev. B 43, 1993 (1991).

[31] J. S. Lin, A. Qteish, M. C. Payne, and V. Heine, Optimized and transferable nonlocal separable ab initio pseudopotentials, Phys. Rev. B 47, 4174 (1993).

[32] S. Nosé, A molecular dynamics method for simulations in the canonical ensemble, Mol. Phys. 52, 255 (1984).

[33] M. Parrinello and A. Rahman, Polymorphic transitions in single crystals: a new molecular dynamics method, J. Appl. Phys. 52, 7182 (1981).

[34] Q. Zeng, Z. He, X. San, Y. Ma, F. Tian, T. Cui, B. Liu, G. Zou, and H.-K. Mao, A new phase of solid iodine with different molecular covalent bonds, Proc. Natl. Acad. Sci. USA 105, 4999 (2008).

[35] See Supplemental Material at http://link.aps.org/supplemental/ 10.1103/PhysRevResearch.3.033174 for further details for the Zeng's model, the numerical data after the Rietveld analysis, and the DFT calculation result of the long-period model.
[36] Superspace group tables by Harold T. Stokes' Website, http:// stokes.byu.edu/iso/ssg.php (accessed 15 July 2021).

[37] Superspace group tables by Akiji Yamamoto's Website, https: //wcp2-ap.eng.hokudai.ac.jp/yamamoto/spgr.html (accessed 15 July 2021).

[38] M. Wu, J. S. Tse, and Y. Pan, Anomalous bond length behavior and a new solid phase of bromine under pressure, Sci. Rep. 6, 25649 (2016).

[39] P. Dalladay-Simpson, J. Binns, M. Peña-Alvarez, M.-E. Donnelly, E. Greenberg, V. Prakapenka, X.-J. Chen, E. Gregoryanz, and R. T. Howie, Band gap closure, incommensurability and molecular dissociation of dense chlorine, Nat. Commun. 10, 1134 (2019).

[40] T. Luty and J. C. Raich, Molecular to atomic transformation in solid iodine under high pressure, Can. J. Chem. 66, 812 (1988).

[41] D. Duan, Y. Liu, Y. Ma, Z. Liu, T. Cui, B. Liu, and G. Zou, Ab initio studies of solid bromine under high pressure, Phys. Rev. B 76, 104113 (2007).

[42] X. San, L. Wang, Y. Ma, Z. Liu, T. Cui, B. Liu, and G. Zou, Theoretical calculations of phase transitions and optical properties of solid iodine under high pressures, J. Phys.: Condens. Matter 20, 175225 (2008).

[43] I. Loa, M. I. McMahon, and A. Bosak, Origin of the Incommensurate Modulation in Te-III and Fermi-Surface Nesting in a Simple Metal, Phys. Rev. Lett. 102, 035501 (2009).

[44] P. H. Svensson and L. Kloo, Synthesis, structure, and bonding in polyiodide and metal iodide-iodine systems, Chem. Rev. 103, 1649 (2003).

[45] M. Węcławik, P. Szklarz, W. Medycki, R. Janicki, A. Piecha-Bisiorek, P. Zieliński, and R. Jakubas, Unprecedented transformation of $\left[\mathrm{I}^{-} \cdot \mathrm{I}_{3}{ }^{-}\right]$to $\left[\mathrm{I}_{4}{ }^{2-}\right]$ polyiodides in the solid state: structures, phase transitions and characterization of dipyrazolium iodide triiodide, Dalton Trans. 44, 18447 (2015).

[46] C. Walbaum, I. Pantenburg, P. Junk, G. B. Deacon, and G. Meyer, Bulky cations and four different polyiodide anions in $\left[\mathrm{Lu}(\mathrm{Db} 18 \mathrm{c} 6)\left(\mathrm{H}_{2} \mathrm{O}\right)_{3}(\text { thf })_{6}\right]_{4}\left(\mathrm{I}_{3}\right)_{2}\left(\mathrm{I}_{5}\right)_{6}\left(\mathrm{I}_{8}\right)\left(\mathrm{I}_{12}\right)$, Z. Anorg. Allg. Chem. 636, 1444 (2010).

[47] D. Tristant, P. Puech, and I. C. Gerber, Theoretical study of polyiodide formation and stability on monolayer and bilayer graphene, Phys. Chem. Chem. Phys. 17, 30045 (2015).

[48] R. J. Husband, I. Loa, K. A. Munro, E. E. McBride, S. R. Evans, H.-P. Liermann, and M. I. McMahon, Incommensurate-toincommensurate phase transition in Eu metal at high pressures, Phys. Rev. B 90, 214105 (2014).

[49] P. Li, X. Du, G. Gao, R. Sun, L. Zhang, and W. Wang, New modulated structures of solid bromine at high pressure, Comput. Mater. Sci. 171, 109205 (2020).

[50] D. Duan, Z. Liu, Z. Lin, H. Song, H. Xie, T. Cui, C. J. Pickard, and M. Miao, Multistep Dissociation of Fluorine Molecules Under Extreme Compression, Phys. Rev. Lett. 126, 225704 (2021).

[51] R. P. Dias and I. F. Silvera, Observation of the WignerHuntington transition to metallic hydrogen, Science 355, 715 (2017).

[52] P. Loubeyre, F. Occelli, and P. Dumas, Synchrotron infrared spectroscopic evidence of the probable transition to metal hydrogen, Nature 577, 631 (2020). 
[53] N. W. Ashcroft, Metallic Hydrogen: A High-Temperature Superconductor? Phys. Rev. Lett. 21, 1748 (1968).

[54] N. W. Ashcroft, Hydrogen Dominant Metallic Alloys: High Temperature Superconductors? Phys. Rev. Lett. 92, 187002 (2004).

[55] S. Azadi, B. Monserrat, W. M. C. Foulkes, and R. J. Needs, Dissociation of High-Pressure Solid Molecular Hydrogen: A Quantum Monte Carlo and Anharmonic Vibrational Study, Phys. Rev. Lett. 112, 165501 (2014).

[56] G. J. Ackland and J. S. Loveday, Structures of solid hydrogen at 300 K, Phys. Rev. B 101, 094104 (2020).

[57] Y. Fujii, K. Hase, Y. Ohishi, H. Fujihisa, N. Hamaya, K. Takemura, O. Shimomura, T. Kikegawa, Y. Amemiya, and T.
Matsushita, Evidence for Molecular Dissociation in Bromine Near 80 GPa, Phys. Rev. Lett. 63, 536 (1989).

[58] H. Fujihisa, Y. Fujii, K. Takemura, and O. Shimomura, Structural aspects of dense solid halogens under high pressure studied by x-ray diffraction-molecular dissociation and metallization, J. Phys. Chem. Solids 56, 1439 (1995).

[59] A. San-Miguel, H. Libotte, J. P. Gaspard, M. Gauthier, J. P. Itié, and A. Polian, Bromine metallization studied by $\mathrm{X}$-ray absorption spectroscopy, Eur. Phys. J. B 17, 227 (2000).

[60] A. San-Miguel, H. Libotte, M. Gauthier, G. Aquilanti, S. Pascarelli, and J.-P. Gaspard, New Phase Transition of Solid Bromine under High Pressure, Phys. Rev. Lett. 99, 015501 (2007). 